Research Article

\title{
Nanomaterial Antibacterial Technology in the Design of Antibacterial Fabrics for Sports Clothing
}

\author{
Yuan Feng \\ Sports Department, University of Shanghai for Science and Technology, Shanghai 200093, China \\ Correspondence should be addressed to Yuan Feng; fengyuan@usst.edu.cn
}

Received 12 August 2021; Revised 26 September 2021; Accepted 16 October 2021; Published 27 October 2021

Academic Editor: Song Jiang

Copyright (C) 2021 Yuan Feng. This is an open access article distributed under the Creative Commons Attribution License, which permits unrestricted use, distribution, and reproduction in any medium, provided the original work is properly cited.

\begin{abstract}
In recent years, nanomaterials have been widely used in sports equipment, and their application in sportswear has also attracted wide attention. This research mainly discusses the application of nanomaterial antibacterial technology in the design of antibacterial fabrics for sports apparel. The method of adding nano- $\mathrm{TiO}_{2}$ to the antibacterial textiles varies with the type of fiber or fabric, and the finishing method also changes accordingly. For chemical fibers, the method of blending spinning is mainly used. By adding nano-antibacterial particles in the spinning solution, the polymer is ejected from the spinneret to make antibacterial fibers. This processing method is characterized by good antibacterial durability. The preparation of antimite finishing agent is mainly the dispersion of nanopowder. The selection of the best dispersant is mainly determined by measuring the change of the volume sedimentation rate of $\mathrm{TiO}_{2}$ after 5 days under different dosage and $\mathrm{pH}$ value of each dispersant. Then, the particle size of nano$\mathrm{TiO}_{2}$ and $\mathrm{Ag}$ in different dispersing conditions was tested by TEM, and finally, the best dispersing agent and dispersing process were determined. The optimum of nano-antimite and antibacterial finishing agent is as follows: the dispersing agent is TW-80, the amount of anhydrous ethanol in dispersing medium is 5 times that of powder, the amount of dispersing agent is 1.5 times that of powder, the $\mathrm{pH}$ value is 7 , and the stirring time is not less than $30 \mathrm{~min}$. In the preparation of nano-antimite and antibacterial finishing agent, nanomaterials were used. The weight of nano- $\mathrm{TiO}_{2}$ powder was 20 grams and that of Ag was 20 grams. The dispersion medium (absolute ethanol) was $100 \mathrm{~mL}$ of dispersant. The amount of TW-80 was $30 \mathrm{~g}$, the amount of citric acid was $60 \mathrm{~g}$, the emulsion was very stable, and there was only a small amount of precipitation after being static for 5 days. Considering the characteristics of silk knitted fabric, this experiment uses the exhaustion method in the finishing process to finish the silk knitted fabric. After 50 times of washing, the antibacterial rate of Staphylococcus aureus and Escherichia coli reached 95.13\% and 87.44\%, respectively. This study provides guidance for the application of nanomaterials in the antibacterial field of sports clothing.
\end{abstract}

\section{Introduction}

With the continuous improvement of people's living standards, people began to develop functional products that can inhibit the reproduction of microorganisms or kill bacteria, that is, fibers and textiles that can prevent bacteria and odors. Nanomaterials are widely used. For traditional industries such as textile and light industry, the development of nanomaterials is relatively late, but people's trust and attention to nanomaterials make more and more new nanoproducts in traditional industries continue to launch. In particular, in businesses and enterprises, to be invincible in the fierce market competition, it is necessary to take two methods: one is to continuously improve the performance and price of old products, and the other is to innovate constantly and develop new products with new features [1].

With the improvement of people's living standards, the pursuit of quality of life tends to be environmentally friendly and healthy, so new antibacterial clothing fabrics are widely used. In this study, nano- $\mathrm{Ag} / \mathrm{TiO}_{2}$ inorganic antibacterial powder with both advantages was selected to finish cotton fabric, and a kind of cotton fabric with antibacterial function was studied, which can be not only used as sports clothing fabric, but also widely used as medical substrate. It provides theoretical and experimental basis for the application of the antibacterial powder in the textile field.

Yanyan et al. believe that multifunctional carbon-based nanomaterials (MCBNs) with unparalleled optical, 
electronic, and thermal properties have attracted more and more interest and show the greatest promise in the field of biomolecular imaging and therapy. Therefore, carbon-based nanomaterials have a large number of potential applications in the field of biomedicine, such as bioimaging, drug delivery, and tumor treatment. This research only stayed on the theoretical level and did not use experiments for exploration [2]. Sustainable and cost-effective energy production has become the key to meeting current energy needs. Paul et al. believe that inexpensive, scalable, efficient, and reliable catalysts must be developed. This research only presented the description of $3 \mathrm{D}$ porous carbon electrode, and the research process is not very clear [3]. Carbon-based nanomaterials have been developed for photothermal cancer treatment, but it is still a huge challenge to manufacture their multifunctional counterparts with simple methods, good biocompatibility and dispersion, and efficient cancer treatment methods. Li et al. have developed an alternative multifunctional nanoplatform based on carbon silica nanocapsules with gold nanoparticles (Au@CSN) in the cavity for cancer treatment. The encapsulated chemical doxorubicin can be released from Au@CSN with mesoporous and hollow structure by means of near-infrared light and $\mathrm{pH}$ stimulation. Although this research is conducive to spatiotemporal therapy to reduce off-target toxicity, the research method is too cumbersome [4]. Nanocapsules with efficient photothermal conversion and excellent biocompatibility realize the synergistic effect of photothermal and chemotherapy. Exercise under hypoxic conditions represents the extra stress associated with normoxic exercise. Santos et al. believe that hypoxia can induce oxidative stress inflammation, which may be exacerbated after exercise. In addition, vitamin $\mathrm{E}$ supplementation may reduce oxidative stress and inflammation caused by hypoxia during exercise. They evaluated the effect of vitamin E supplementation (250 mg) on inflammatory parameters and cell damage after hypoxic exercise at a simulated altitude of $4200 \mathrm{~m}$. Nine volunteers were supplemented with vitamin E 1 hour before exercise, and performed three 60-minute exercises (with maximum oxygen uptake being 70\%) under normoxia, hypoxia, and hypoxia + vitamin, interspersed for 1 week. Blood was collected before exercise, immediately after exercise, and 1 hour after exercise to measure inflammatory parameters and cell damage. This research only judged the influence of oxygen content on physical health and did not analyze the exercise time in detail [5].

Other research mainly discusses the application of nanomaterial antibacterial technology in sports apparel [6]. The preparation of antimite finishing agent is mainly the dispersion of nanopowder. Then, the particle sizes of nano$\mathrm{TiO}_{2}$ and $\mathrm{Ag}$ of the determined optimal dispersant under different dispersing process conditions were tested by transmission electron microscopy (TEM), and the best dispersant and dispersion process were finally determined. The best process of nano-antimite and antibacterial finishing agent is as follows: the dispersant is TW-80, the amount of dispersion medium containing anhydrous ethanol is 5 times the amount of powder, the amount of dispersant is 1.5 times the amount of powder, the $\mathrm{PH}$ value is 7 , and the mixing time is not less than $30 \mathrm{~min}$. The preparation of nanoantimite and antibacterial finishing agent adopts the method of nanomaterial compounding: 20 grams of nano- $\mathrm{TiO}_{2}$ powder, 20 grams of $\mathrm{Ag}, 100 \mathrm{~mL}$ of dispersion medium (absolute ethanol), $30 \mathrm{~g}$ of dispersant TW-80, and $60 \mathrm{~g}$ of citric acid. The prepared emulsion is very stable after standing for 5 days with only a small amount of precipitation. Considering the characteristics of silk knitted fabrics, in this experiment, the exhaustion method is used in the finishing process to finish the silk knitted fabrics.

\section{Nanomaterial Antibacterial Effects}

2.1. Nanomaterials. The full dispersion of nanoparticles is the key and difficult point of the research, which directly gives full play to the antibacterial and antimite effects of nanoparticles. This is the problem to be solved in this experiment [7]. In practical application, there are many factors that affect the full dispersion of nanoparticles, such as mixing method, mixing time, and dispersion medium, as well as the influence of temperature and humidity of the test environment and human factors, so it is key in the preparation of antimite finishing agent to determine the best dispersion process by a large number of repeated experiments $[8,9]$. The pore size of nanomaterials is expressed as follows:

$$
E g_{o s}-E g_{r e f}=\Delta E g \approx \frac{h^{2} \pi^{2}}{2 R^{2}} \times \frac{1}{\mu}-\frac{1.8 e^{2}}{\varepsilon R}
$$

Among them, $E_{g_{\text {ref }}}$ represents the initial potential energy of the nanoparticle, $R$ is the longest radius, and $\mu$ is the corresponding particle size coefficient. The surface coefficient of nanoparticles will change correspondingly during the preparation process [10].

$$
\begin{aligned}
c_{\tau} & =\tau_{0}+\alpha g b \rho^{1 / 2}=\tau_{0}+\alpha g b \rho^{p-N}, \\
\tau & \left.=\left[\frac{G b}{2 \pi L(1-v)}\right]\left\{1-\frac{v}{2}\left(3-4 \cos ^{2} \phi\right)\right] \ln \left(\frac{L}{\eta}\right)-1+\frac{V}{2}\right\} .
\end{aligned}
$$

Among them, $c_{\tau}$ is the surface coefficient, and $\tau_{0}$ is the value of the initial strain force. After that, other forms of micromechanical peeling were obtained. This improves the quality and quantity of multifunctional nanomaterials prepared by micromechanical peeling to various degrees in terms of the number of control layers or the improvement of the edge structure $[11,12]$. An organic film can be formed by adding a corresponding physical coating on the surface of the nanoparticles. The organic film sensitivity value is expressed as follows:

$$
m=\frac{\sqrt{3} k_{B} T}{a G b^{3}}\left[\ln \left(\frac{V}{b^{3}}\right)-1.63\right]^{-1}+\rho V .
$$

Among them, $m$ represents the event sensitivity coefficient, and $k_{B}$ is the correlation value of the sensitivity coefficient. Due to the small diameter of the nanoparticle, the surface area increases sharply, the surface energy is higher, 
and the number of surface atoms is far more than that of non-nanomaterials of the same mass. The activity of nanomaterials is expressed as follows [13]:

$$
V \%=\frac{[A]_{\text {sample }}-[A]_{\text {blank }}}{[A]_{\text {positivecontrol }}-[A]_{\text {blank }}} \times 100 \% .
$$

Among them, $[A]_{\text {sample }}$ is the quality of the sample, and $[A]_{\text {blank }}$ is the quality of the blank control. Nanomaterial carriers can improve the transparency of biological cell membranes and the compatibility of compounds $[14,15]$.

$$
\begin{aligned}
& E E \%=C_{\text {sample }} \times \frac{V}{M_{\text {drug }}} \times 100 \%+C_{\text {sample }} \times V_{\text {min }}, \\
& D L \%=C_{\text {sample }} \times \frac{V}{\left(C_{\text {sample }} \times V+M_{\text {micelle }}\right)} \times 100 \% .
\end{aligned}
$$

Here, $C_{\text {sample }}$ is the DOX concentration measured with the nanoparticles loaded with drugs and the osmotic pressure value of $E E \%$ nanoparticles [16].

2.2. Nano-Antibacterial Effects. The antimite and antibacterial research of nanotextiles is still in the further improvement stage. The particle sizes of nanomaterials on cotton knitted garments are all greater than 150 nanometers, beyond the basic requirement of nanomaterials that the particle size should be less than 100 nanometers in order to give full play to the characteristics of nanomaterials, so the antibacterial ability is low, with no resistance to washing, mainly because of the nanomaterials. They are not organically combined with the cotton fiber raw materials in knitted garments, so the washing resistance is low, and they can only be washed less than 10 times, which has no practical value $[17,18]$. Therefore, the research and development of practical nano-mite-proof and antibacterial cotton knitted garments have become an urgent matter, which has greater practical significance and wider market application prospects. At the same time, it is also the focus of this research to ensure that the finished clothing has excellent antimite and antibacterial properties. The washing fastness of the nano-antimite and antibacterial finishing agent is also the focus of this research. The mixture is used to ensure the washing fastness of antimite and antibacterial clothing with no changes in its various properties (such as air permeability, skin affinity, strength, feel, and color). Staphylococcus aureus and Escherichia coli are shown in Figure 1.

The amount of information transmitted by nanomaterials to signals is KL [19]; namely,

$$
K L=-\log _{2} P\left(L_{i}\right) \text {. }
$$

Then, the information entropy is [20]

$$
H(L)=-\sum_{i=1}^{n} P\left(L_{i}\right) \log _{2} P\left(L_{i}\right) .
$$

The research and development of functional textile products with antibacterial properties, while maintaining the good feel and wearability of the fabric itself, not only are a requirement for technology, but also conform to the needs of contemporary people for the health function of textile products. While preventing diseases, this is consistent with people's promotion of healthy and relaxed lifestyles. In addition, there is a signal exchange between information $X$ and $Y$; then [21],

$$
\begin{aligned}
& R\left(\frac{X}{Y}\right)=-\sum_{i=1}^{n} \sum_{j=1}^{m} M\left(X_{i} Y_{j}\right) \log _{2} N\left(\frac{X_{i}}{Y_{j}}\right), \\
& R P\left(t_{j}\right)=\sqrt{\frac{1}{n} \sum_{i=1}^{n}\left[T P\left(t_{j}\right)-H P_{i}\left(t_{j}\right)\right]^{2}} .
\end{aligned}
$$

Among them, $t_{j}$ is the time of the current processing period, and $n$ is the total number of items remaining after the previous processing. Polyethylene (lactic acid) is copolymerized with mesoporous multifunctional nanomaterials [22]. The organic film sensitivity value is expressed as follows:

$$
V=\frac{k_{B} T}{A G B^{3}}\left[\ln \left(\frac{V}{B^{3}}\right)-2\right]^{-1}+\rho V .
$$

Among them, $V$ represents the sensitivity coefficient. In the study of conduction rate, resistance is a frequently considered issue $[23,24]$.

$$
Z_{\text {in }}=\sqrt{\frac{\mu_{r}}{\varepsilon_{r}^{3}}} \tanh \left[-j\left(\frac{2 \pi}{c}\right)\right]+\left(\sqrt{\frac{\mu_{r}}{\varepsilon_{r}}} f d\right) \sum_{i=1}^{n} M .
$$

When the correlation coefficient $Z_{\text {in }}$ of resistance is constant, the size of the conductivity depends on the radius of a single molecule. In the use of related nanomaterials, it is usually necessary to consider the quality of its energy absorption $R_{L}(\mathrm{~dB})$ [25].

$$
R_{L}(\mathrm{~dB})=-20 \log _{10}\left[\left|\left(Z_{\text {in }}-1\right)\left(Z_{\text {in }}+1\right)\right|\right] \sum_{i=1}^{n} \frac{M}{Z_{1} Z_{2}} .
$$

Information processing is carried out in sections, and each section needs to be refined. The absorption of energy can indirectly show the performance of related materials. After the preparation of nanomaterials, the concept of corresponding coefficients needs to be introduced at this time [26].

$$
Q_{g}=\frac{\Delta P}{P_{a}-\Delta P}+\sum_{i=1}^{N}\left(Z_{M}-Z_{N}\right)\left(Z_{2}-Z_{1}\right) .
$$

Keep the conduction rate $Q_{g}$ unchanged, and when the change value of the air pressure increases, the sensitivity performance of the nanomaterials will increase accordingly. The mechanical properties of nanomaterials largely depend on the length of the diameter [27].

$$
\begin{aligned}
\varepsilon_{N H} & =A_{N H} \frac{D \Omega \sigma}{K T d^{2}}\left(Z_{M}-Z_{N}\right) \sum_{i=1}^{n}\left(D_{2}-D_{1}\right)\left|X_{M}+X_{N}\right|, \\
\varepsilon_{c 0} & =\frac{D_{G b} \Omega \delta \sigma}{K T d^{3}} \sum_{i=1}^{n}\left(Z_{M}-Z_{N}\right)\left|X_{2}-X_{1}\right| .
\end{aligned}
$$


When the conductivity is constant, the change between the maximum value $\varepsilon_{N H}$ and the minimum value $\varepsilon_{c 0}$ of the diameter has the most obvious effect on the stress change of the nanomaterials $[28,29]$.

$$
M_{k}(T)=\sum_{i=1}^{N} u_{k}(T) \times \delta\left(T-\tau_{1}\right) \times p_{k}(T) \times g_{k i}(T) \times M .
$$

In information transmission, signal transmission usually requires a certain process, and the transmission cycle $T$ will also be affected by related factors [30].

$$
W_{p}(t)=\sum_{k=1}^{M}\left[W_{k}(t) \times l_{k}(t)\right]=\sum_{k=1}^{M}\left[\sum_{i=1}^{M} \delta\left(t-t_{k i}\right)\right]\left(t_{k}+k_{i}\right) .
$$

The potential energy of repulsion when the attached layer is compressed is

$$
U_{R}^{e}=N_{S} K T \theta \infty\left(\frac{1-h}{l}\right)
$$

where $N_{s}$ represents the number of molecules adsorbed per unit area; $\theta \infty$ represents the extent to which the surface is covered by molecules when $H=\infty$; and $H$ represents the distance between the two surfaces close to each other $[31,32]$.

\section{Nano-Antibacterial Sportswear Experiment}

3.1. Preparation of Nano-Antimite Finishing Agent. The preparation of antimite finishing agent is mainly the dispersion of nanopowders. The full dispersion of nanoparticles is related to factors such as $\mathrm{pH}$ value, dispersant dosage, stirring method, and dispersion medium, so in the dispersion experiment, mainly start from the above aspects. To solve the dispersion problem, the experiment is mainly divided into two parts: the first is the choice of dispersant. This is because there are many types of dispersants, and an optimal dispersant that can fully disperse the nanopowder must be selected. The second is determining the best dispersant. Dispersant experiments were carried out to get the best dispersion process.

3.1.1. Design Ideas. The following design ideas were considered: retrieval of related literature $\longrightarrow$ design experimental plan $\longrightarrow$ experiment $\longrightarrow$ best experimental plan $\longrightarrow$ experiment $\longrightarrow$ several dispersants with relatively good dispersion effect $\longrightarrow$ experiment $\longrightarrow$ best dispersant and dispersion medium $\longrightarrow$ experiment $\longrightarrow$ determining the best dispersion process.

3.1.2. Experimental Instruments and Reagents. The following experimental instruments and reagents were used:

BME100LX high-shear mixing emulsifier (Shanghai Weiyu Electromechanical Manufacturing Co., Ltd.).
TEM-200CX transmission electron microscope (School of Materials Science) (the main reagent information is shown in Table 1).

Nano- $-\mathrm{TiO}_{2}$ (particles of $50-80 \mathrm{~nm}$ ) (Jiangsu Changtai Nanometer Material Co., Ltd.).

Nano-Ag (particle size of $20 \mathrm{~nm}$ ) (Beijing Saiterui Nano Co., Ltd.).

The selection of the above dispersants is based on the dispersion theory and initial preliminary experiments. Among the above several dispersants, citric acid, SDS, SDBS, and SD-03 are ionic dispersants, mainly based on the electric double layer theory for nanometer. The particles are dispersed. PEG, TW-80, and OP are nonionic dispersants, which are mainly based on the theory of steric stability to disperse nanoparticles.

Experimental steps are as follows: weigh $\mathrm{Ag}$ and $\mathrm{TiO}_{2} \longrightarrow$ add $\quad$ water $\longrightarrow$ stir $\longrightarrow$ add dispersant $\longrightarrow$ stir $\longrightarrow$ adjust $\quad \mathrm{PH}$ value $\longrightarrow$ stir $\longrightarrow$ stand still $\longrightarrow$ observe the dispersion effect.

3.2. Best Dispersant and Dispersion Process Selection. The choice of the best dispersant is mainly determined by measuring the change in the volume sedimentation rate of $\mathrm{TiO}_{2}$ after 5 days of standing for each dispersant under different dosage and $\mathrm{pH}$ conditions. Then, the determined optimal dispersant and the nanometer $\mathrm{TiO}_{2}$ and Ag particle size under different dispersing process conditions were tested by TEM, and the best dispersant and dispersion process were finally determined.

3.2.1. Selection of Ionic Dispersant. Disperse SDS, SDBS, and SD-03 ionic dispersants according to the process in Table 1, then put them in a graduated cylinder under the same conditions, let them stand for 5 days, and then calculate the sedimentation rate relative to the entire system. The smallest value corresponds to the dispersant and dispersant dosage.

The minimum sedimentation rate of the dispersant SDS is $0.045 \%$, the corresponding dispersant dosage is $0.5 \%$, and the minimum sedimentation rate of the dispersant SDBS is $0.046 \%$, which is similar to the minimum sedimentation rate of the dispersant SDS, which corresponds to the dispersion. The dosage of the dispersant is $0.2 \%$, the dispersion effect of the dispersant SD-03 is the worst, and its minimum sedimentation rate is $0.086 \%$, which corresponds to a dispersant dosage of $1 \%$. Change the $\mathrm{PH}$ value of the above dispersant at its optimal dosage, and also put it for 5 days to observe the volume sedimentation rate.

Among the three ionic dispersants used in the experiment, the dispersant SDBS had the best dispersion effect, the best dosage was $0.2 \%$, and the best $\mathrm{PH}$ value was 10 ; the worst dispersion effect was that of the dispersant SD-03. The particle dispersion effect is best when $\mathrm{pH}=10$.

The above is the selection of the best ionic dispersant. Use the same experimental method to select the best nonionic dispersant, then compare the dispersion effect of the 

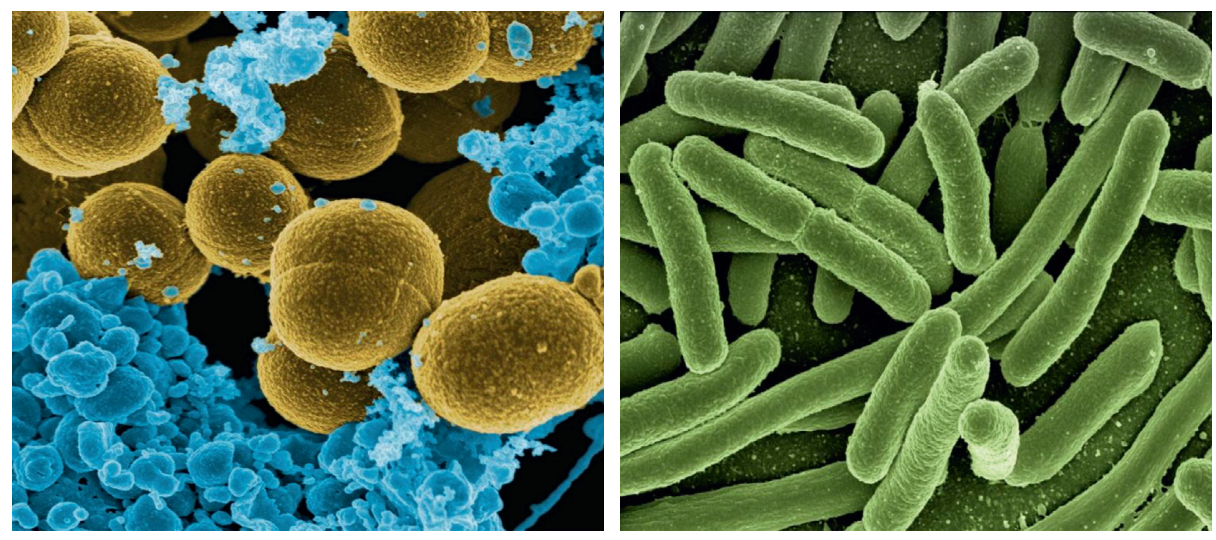

FIgURE 1: Staphylococcus aureus and Escherichia coli (http://alturl.com/uc3j5 and http://alturl.com/fmpiu).

TABLE 1: Main reagent information.

\begin{tabular}{|c|c|c|}
\hline Drug name & Analytically pure & Factory \\
\hline Citric acid & Analytically pure & Bengbu Chemical Reagent Factory \\
\hline SDS & Chemically pure & Hedong District Hongyan Chemical Reagent Factory \\
\hline SDBS & - & Yifa Chemical Reagent Factory \\
\hline SD-03 & Chemically pure & A chemical research institute \\
\hline PEG & Chemically pure & Kemeou Chemical Reagent Development Center \\
\hline TW -80 & Chemically pure & Zonghengxing Industry and Trade Co., Ltd., Chemical Reagent Company \\
\hline $\mathrm{OP}$ & Analytically pure & Yifa Chemical Reagent Factory \\
\hline Absolute ethanol & Analytically pure & Chemical reagent factory \\
\hline Ethylene glycol & Analytically pure & Zongheng Xinggong Chemical Reagent Company \\
\hline
\end{tabular}

ionic dispersant and nonionic dispersant, and finally select the best dispersant and dispersion process.

3.2.2. Selection of Nonionic Dispersant. The dispersing principle of nonionic dispersant is to form an adsorption layer on the surface of the particles, so that the particles are dispersed. Therefore, the amount of dispersant is much larger than that of particle-type dispersants, and it is no longer nanopowder. Furthermore, its $\mathrm{pH}$ value is no longer the main influencing factor, so the $\mathrm{pH}$ value is set to 7 in the experiment.

Comparing the volume sedimentation rate of PEG, TW80 , OP, and nonionic dispersant, we can find that the overall volume sedimentation rate of dispersant TW-80 is very small, the smallest can reach $0.015 \%$, and the corresponding dispersant dosage is 1.5 times the amount of nanopowder. Combining the above dispersants, we can find that the best dispersing effect is the nonionic dispersant TW-80, and its optimum dosage is 1.5 times the amount of nanopowder. It can be seen from the TEM photo that the dispersant TW-80 has the best dispersion effect when the amount of dispersant is 1.5 times the amount of $\mathrm{TiO}_{2}$.

3.2.3. Determination of $p H$. The best process of nanoantimite and antibacterial finishing agent is as follows: the dispersant is $\mathrm{TW}-80$. The $\mathrm{pH}$ value is 7 , and the mixing time is not less than $30 \mathrm{~min}$.
3.3. Nano-Antimite and Antibacterial Finishing Agent. In order to achieve the expected goal, i.e., to kill bacteria and repel mites and to decompose their residual nuclei and remains, we used the way of nanomaterials compounding. Nano-Ag is a contact antibacterial agent, which kills the bacteria by destroying the sulfhydryl group of the enzyme protein in the bacteria by the free $\mathrm{Ag}+\mathrm{Nano}-\mathrm{TiO}_{2}$ is a photocatalytic antibacterial agent. It kills bacteria and decomposes organic matter by decomposing various bacteria or organic matter into small inorganic molecules such as $\mathrm{CO}_{2}$ and $\mathrm{H}_{2} \mathrm{O}$ through the highly oxidizing $\mathrm{HO} \bullet$ and $\bullet \mathrm{O}_{2}$.

The weight of nano- $\mathrm{TiO}_{2}$ powder is 20 grams and $\mathrm{Ag}$ is 20 grams, the volume of dispersion medium (absolute ethanol) is $100 \mathrm{~mL}$, the amount of dispersant TW-80 is $20 \times 1.5=30 \mathrm{~g}$, the amount of citric acid is $20 \times 3=60 \mathrm{~g}$, and $\mathrm{pH}=7$. Stirring time is $30 \mathrm{~min}$, and stirring speed is $6000 \mathrm{r} /$ min. The emulsion prepared is very stable, and there is only a small amount of precipitation after standing for 5 days. The TEM photos of nano- $\mathrm{TiO}_{2}$ and $\mathrm{Ag}$ under the best dispersion process are shown in Figure 2.

\subsection{Nanometer Antimite and Antibacterial Silk Knitted Garment Finishing}

3.4.1. Finishing Method. For natural fibers, since functional powder cannot be added in the fiber production process, only postprocessing methods can be used. Processing techniques include exhaustion method, padding method, 


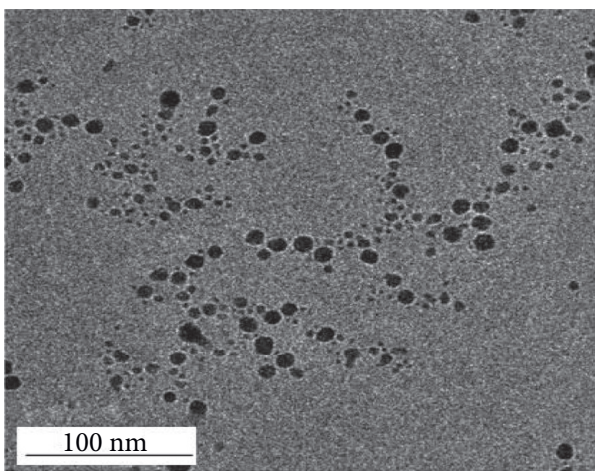

Ag

(a)

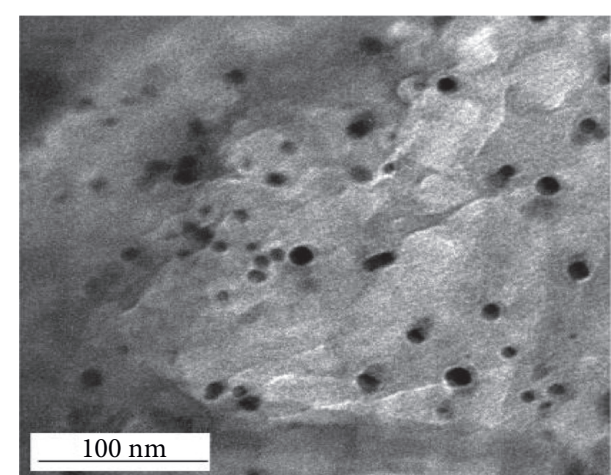

$\mathrm{TO}_{2}$

(b)

FIgURE 2: TEM images of nano- $\mathrm{TiO}_{2}$ and $\mathrm{Ag}$ under the best dispersion process (from http://alturl.com/7j8ad).

and coating method. Considering the characteristics of the silk knitted fabric itself, in this experiment, the exhaustion method is used in the finishing process to finish the silk knitted fabric as follows:

(1) Laboratory apparatus: IR12P infrared dyeing instrument, LD-3624 sample shaping dryer

(2) Medicine: adhesive (Shanghai Fujikawa Chemical Co., Ltd.), detergent: German soap (MT9906017)

(3) Fabric: silk knitted fabrics of various colors (Zhejiang Xinyang Knitting Apparel Company)

(4) Process formula is as follows:

\#1 finishing solution formula: compound finishing dosage $0.5 \% ; \mathrm{TiO}_{2}: \mathrm{Ag}$, respectively, $1: 0,1: 1,1: 2$, $1: 5,1: 10,0: 1$; adhesive $4 \%$; liquor ratio $1: 10$.

\#2 finishing solution formula: compound finishing dosage $1 \% ; \mathrm{TiO}_{2}: \mathrm{Ag}$, respectively, $1: 0,1: 1,1: 2,1$ : $5,1: 10,0: 1$; adhesive $4 \%$; liquor ratio $1: 10$. \#3 finishing solution formula: composite finishing dosage $2 \% ; \mathrm{TiO}_{2}: \mathrm{Ag}$, respectively, $1: 0,1: 1,1: 2,1$ : $5,1: 10,0: 1$; adhesive $4 \%$; liquor ratio $1: 10$.

3.4.2. Process Flow. The process flow is summarized as follows: fabric washing $\longrightarrow$ dehydration $\longrightarrow$ immersion of the fabric in a dyeing cup filled with finishing liquid$\longrightarrow$ heating up (reaching $40^{\circ} \mathrm{C}$ within $10 \mathrm{~min}$ ) $\longrightarrow$ keeping warm $(30 \mathrm{~min}) \longrightarrow$ prebaking at $90^{\circ} \mathrm{C}$ for $5 \mathrm{~min} \longrightarrow$ baking at $120^{\circ} \mathrm{C}$ for $2 \mathrm{~min} \longrightarrow$ washing.

3.4.3. Washing Method. Follow the Japanese Industrial Standards (JIS) washing method. Use household alkaline detergents (polyoxyethylene alkyl ether and sodium $\alpha$-olefin xanthate, 9:1 compound) in household washing machines with a bath ratio of $1: 30$. Washing at $40^{\circ} \mathrm{C}$ for 5 minutes and two washings with water for 2 minutes at room temperature constitute a cycle.

\section{Discussion}

4.1. Bacteriostatic Test Analysis. The effect of different composite finishing agents is shown in Figure 3, with different amounts of composite finishing agent: $0.5 \%, 1 \%$, and $2 \%$. Taking into account the high price of nanopowder, from the perspective of enterprise cost, the ratio of $\mathrm{TiO}_{2}$ and $\mathrm{Ag}$ is $1: 1,1$ : 2 , and $1: 3$, respectively, to finish the fabric, and then the shaking flask method is used to carry out the antibacterial test.

When the dosage of the composite finishing agent is $0.5 \%$, the $\mathrm{TiO}_{2}: \mathrm{Ag}$ ratio is $1: 3,1: 2$, and $1: 1$, and the finished fabrics are numbered \#1, \#2, and \#3. That is to say, the household alkaline detergent polyoxyethylene alkyl ether and $\alpha$-olefin yellow sodium are compounded in a ratio of 9 : 1. After 20 washings, the antibacterial effect was measured by the shaking flask method. After shaking for 5 hours by the shaking flask method, the antibacterial situation of the finished fabric is shown in Table 2.

During the test, when the ratio of $\mathrm{TiO}_{2}$ to $\mathrm{Ag}$ is $1: 3$, the antibacterial effect is the best, reaching more than $94 \%$. After washing the fabric 20 times, the antibacterial effect on gold can be stabilized above $97 \%$. By gradually narrowing the scope of the method, the final determination of the best process is as follows: the amount of composite finishing agent is $0.5 \%$, and the ratio of $\mathrm{TiO}_{2}$ and $\mathrm{Ag}$ is $1: 3$. The antibacterial situation of the fabric after 20 washings is shown in Table 3 .

In the study, the sample "nano-mite-proof silk knitted garment fabric" (after washing 50 times) was used for 6 hours, and the antibacterial rate against Staphylococcus aureus (ATCC6S38) reached more than 50.0\%; the sample "nano-mite-proof silk knitted garment fabric" (after washing 50 times in water) acts for 6 hours, and its antibacterial rate against Escherichia coli reaches over $50.0 \%$. The antibacterial effect of "nano-mite-proof silk knitted garment fabric" on Staphylococcus aureus is shown in Table 4.

Under the condition of the test temperature of $20 \pm 2^{\circ} \mathrm{C}$, the nanometer antimite silk knitted garment fabrics submitted for inspection have been used for 6 hours, and the antibacterial rate against Staphylococcus aureus (ATCC6538) 

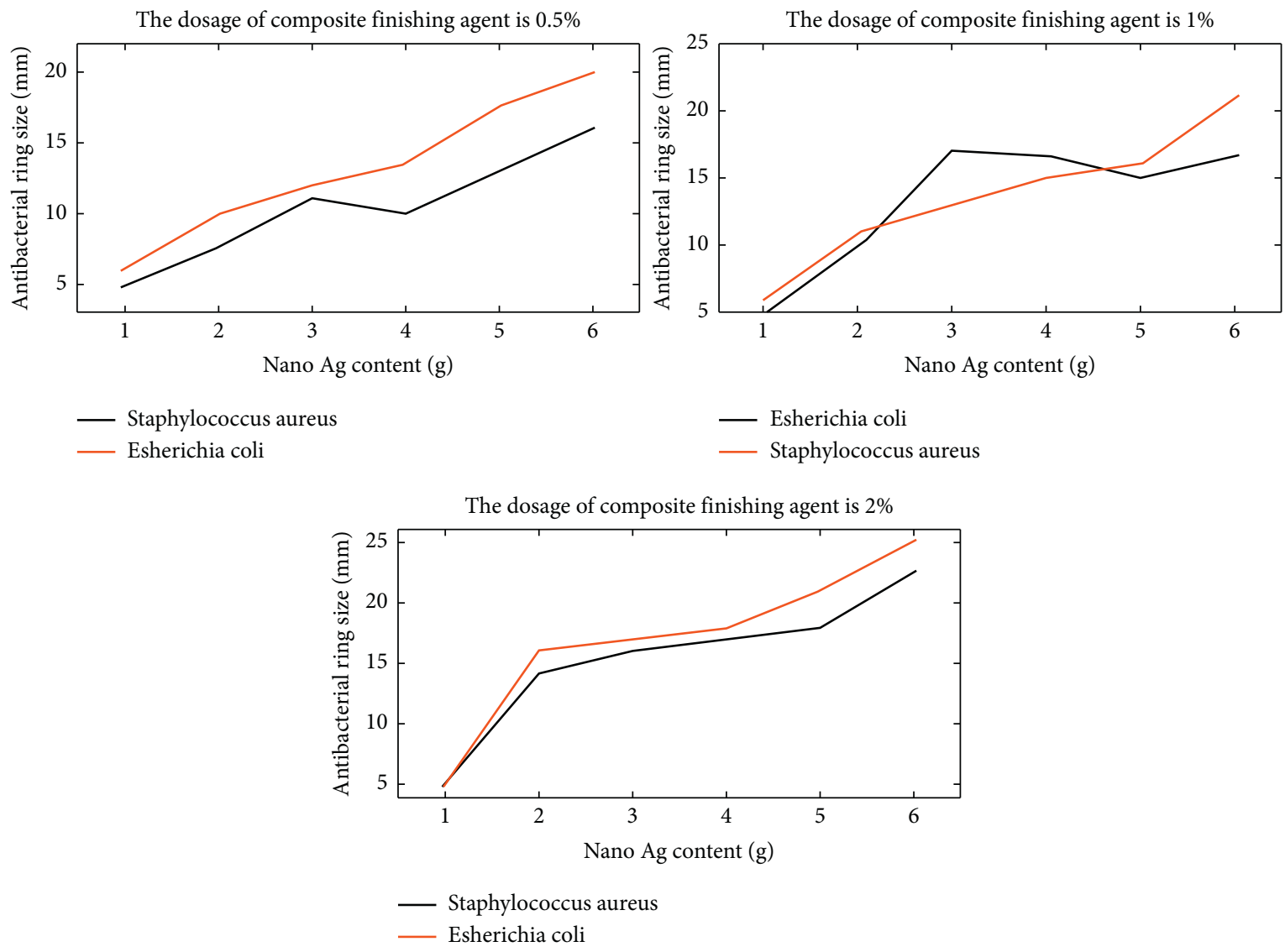

FIgURE 3: Different amounts of composite finishing agent.

is above $90 \%$. The antibacterial effect of "nano-mite-proof silk knitted garment fabric" on Escherichia coli is shown in Table 5.

4.2. Fabric Performance Test Analysis. During the test, each sample is tested 10 times, and then the average value is calculated. The area of presser foot is $100 \mathrm{~mm}^{2}$, the diameter of presser foot is $10.28 \mathrm{~mm}$, the pressure is $200 \mathrm{~N}$, and the pressure time is $10 \mathrm{~s}$. It can be seen from the data of the average diameter of the contrast samples in Figure 4 that the thickness of the fabric before and after finishing does not change at all. The comparison of the average diameter of the fabric thickness is shown in Figure 4.

Air permeability is an important indicator of the comfort of wearing clothing. The finished fabric and the original sample are tested on 5 samples, and the average value is calculated. It can be seen that the air permeability of the finished fabric is the same or slightly better. The air permeability comparison is shown in Figure 5.

The better the drape of the fabric, the better the material. It can be seen from Figure 6 that the drape of the finished fabric is better than the original or remains unchanged, and there is no sample with worse drape than the original. The drape of the fabric is shown in Figure 6.

Breaking strength and elongation at break are important indexes reflecting the mechanical properties of fabrics. It can be seen from Table 6 that there is no obvious change in the indexes of fabric strength before and after finishing; that is to say, the antibacterial and antimite fabric developed in this study has little effect on bursting strength and tensile length. The strength of reactive fabric is shown in Table 6 .

The difference between this experiment and the previous experiment is that this experiment is conducted to deal with the clothing, not the piece of clothing, so there may be some problems that did not appear in the sample experiment. In the experiment, we try to choose different colors, different raw materials, and different fabrics for the selection of silk knitwear. The purpose is to verify the antibacterial properties of different materials and the impact on their properties and lay the foundation for the next batch production of enterprises. Different clothing properties are shown in Table 7.

Before and after finishing, the experiment condition temperature in the constant temperature box is $25 \pm 1^{\circ} \mathrm{C}$, and the relative humidity of the dryer is $75 \pm 2 \%$. Nanoantimite and antibacterial knitted garments have an effect of 10 minutes, and the antibacterial rate against Staphylococcus aureus (ATCC6538) reaches more than 99.04\%. After washing 50 times, the antibacterial rate against Staphylococcus aureus (ATCC6538) reached over 95.13\%, and the antibacterial rate against Escherichia coli (8099) reached over $87.44 \%$. The antibacterial effect of nanometer antimite and antibacterial knitted garments is shown in Figure 7. 
TABLE 2: The antibacterial situation of the finished fabric after shaking for 5 hours by the shaking flask method.

\begin{tabular}{|c|c|c|c|c|c|}
\hline \multirow{3}{*}{ Bacteria } & \multirow{3}{*}{$\begin{array}{c}\text { Swatch } \\
\text { Numbering } \\
\text { Original sample }\end{array}$} & \multicolumn{2}{|c|}{$\begin{array}{l}\text { Diluent and bacterial } \\
\text { concentration (cfu/tablet) }\end{array}$} & \multirow{3}{*}{$\begin{array}{c}\text { Bacteria concentration } \\
\text { (cfu/mL) } \\
5.23 \times 10^{5}\end{array}$} & \multirow{3}{*}{$\begin{array}{c}\text { Inhibition rate } \\
(\%) \\
-\end{array}$} \\
\hline & & 100 & $10^{-1}$ & & \\
\hline & & Uncountable & Uncountable & & \\
\hline Golden yellow & $\# 1$ & 468 & 48 & $5.22 \times 10^{5}$ & 99.90 \\
\hline \multirow{2}{*}{ Staphylococcus } & $\# 2$ & 491 & 52 & $4.91 \times 10^{5}$ & 99.87 \\
\hline & $\# 3$ & 499 & 53 & $3.94 \times 10^{5}$ & 97.48 \\
\hline Large intestine & $\# 1$ & Uncountable & 462 & $3.57 \times 10^{5}$ & 97.26 \\
\hline Bacillus & $\# 2$ & Uncountable & 487 & $4.01 \times 10^{5}$ & 96.8 \\
\hline
\end{tabular}

TABle 3: Bacteriostasis of fabric after 20 washings.

\begin{tabular}{|c|c|c|c|c|c|}
\hline \multirow{3}{*}{ Bacteria } & Swatch & \multicolumn{2}{|c|}{$\begin{array}{l}\text { Diluent and bacterial } \\
\text { concentration (cfu/tablet) }\end{array}$} & \multirow{3}{*}{$\begin{array}{c}\text { Bacteria concentration } \\
\text { (cfu/mL) } \\
5.23 \times 10^{5}\end{array}$} & \multirow{3}{*}{$\begin{array}{c}\text { Inhibition rate } \\
\text { (\%) } \\
-\end{array}$} \\
\hline & Numbering & 100 & $10^{-1}$ & & \\
\hline & Original sample & Uncountable & Uncountable & & \\
\hline Golden yellow & $\# 1$ & Uncountable & 348 & $5.23 \times 10^{5}$ & 98.85 \\
\hline \multirow{2}{*}{ Staphylococcus } & $\# 2$ & Uncountable & 452 & $4.82 \times 10^{5}$ & 98.13 \\
\hline & $\# 3$ & Uncountable & 453 & $4.01 \times 10^{5}$ & 98.25 \\
\hline Large intestine & $\# 1$ & Uncountable & Uncountable & $3.59 \times 10^{5}$ & 97.21 \\
\hline Bacillus & $\# 2$ & Uncountable & Uncountable & $3.99 \times 10^{5}$ & 96.74 \\
\hline
\end{tabular}

TABLE 4: Antibacterial effect of nano-antimite silk knitted garment fabrics on Staphylococcus aureus.

\begin{tabular}{|c|c|c|c|}
\hline \multicolumn{4}{|c|}{ The average bacteriostatic rate and range of different action time (\%) } \\
\hline \multirow{5}{*}{ Average number of colonies and range of positive control group (cfu/tablet) } & $1 \mathrm{~h}$ & $6 \mathrm{~h}$ & $12 \mathrm{~h}$ \\
\hline & 33.49 & 54.65 & 64.19 \\
\hline & $(32.99-33.90)$ & $(53.33-55.40)$ & $(63.41-64.88)$ \\
\hline & $5.77 \times 10^{4}$ & $5.37 \times 10^{4}$ & $5.13 \times 10^{4}$ \\
\hline & $4.85 \times 10^{4}-6.66 \times 10^{4}$ & $4.35 \times 10^{4}-6.2 \times 10^{4}$ & $4.10 \times 10^{4}-6.11 \times 10^{4}$ \\
\hline
\end{tabular}

TABLE 5: Antibacterial effect of "nano-mite-proof silk knitted garment fabric" on Escherichia coli.

\begin{tabular}{lccc}
\hline \multicolumn{4}{c}{ The average bacteriostatic rate and range of different action time (\%) } \\
\hline & $1 \mathrm{~h}$ & $6 \mathrm{~h}$ & $12 \mathrm{~h}$ \\
& 85.88 & 91.79 & 94.19 \\
Average number of colonies and range of positive control group & $(85.21-86.64)$ & $(91.50-92.17)$ & $(93.41-94.88)$ \\
(cfu/tablet) & $5.68 \times 10^{4}$ & $5.36 \times 10^{4}$ & $4.13 \times 10^{4}$ \\
& $4.5 \times 10^{4}-6.69 \times 10^{4}$ & $4.25 \times 10^{4}-6.38 \times 10^{4}$ & $3.10 \times 10^{4}-5.11 \times 10^{4}$ \\
\hline
\end{tabular}

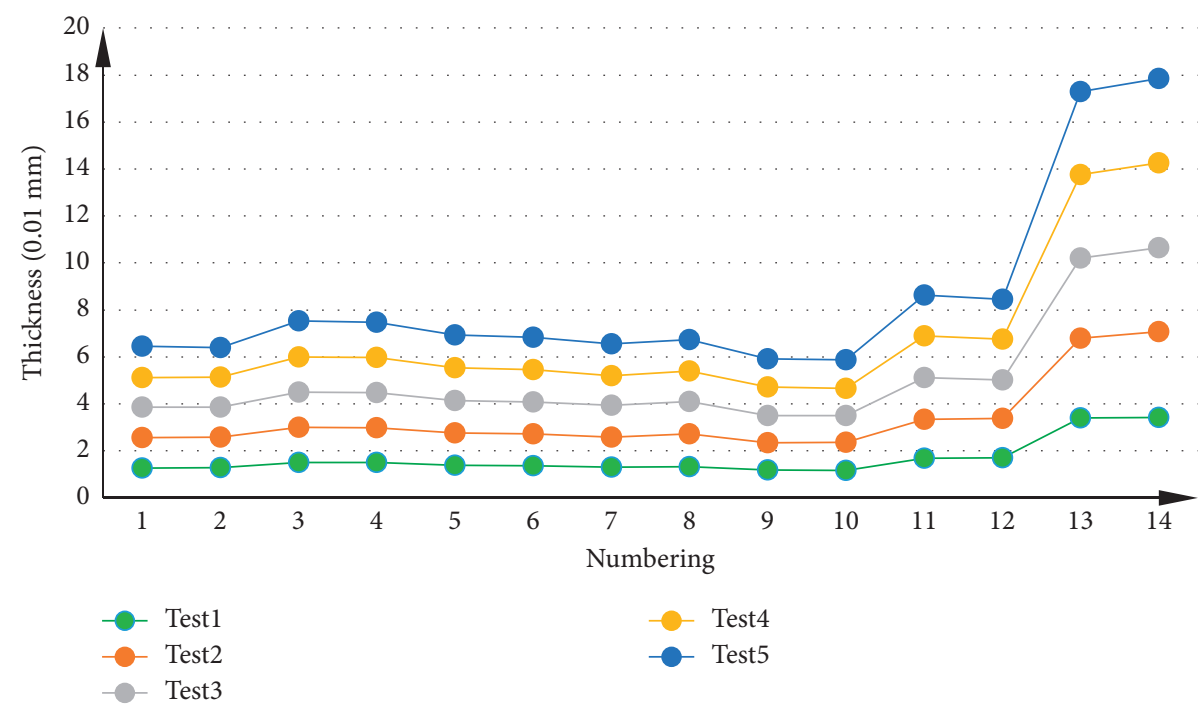

FIgURE 4: Comparison of average diameter of fabric thickness. 


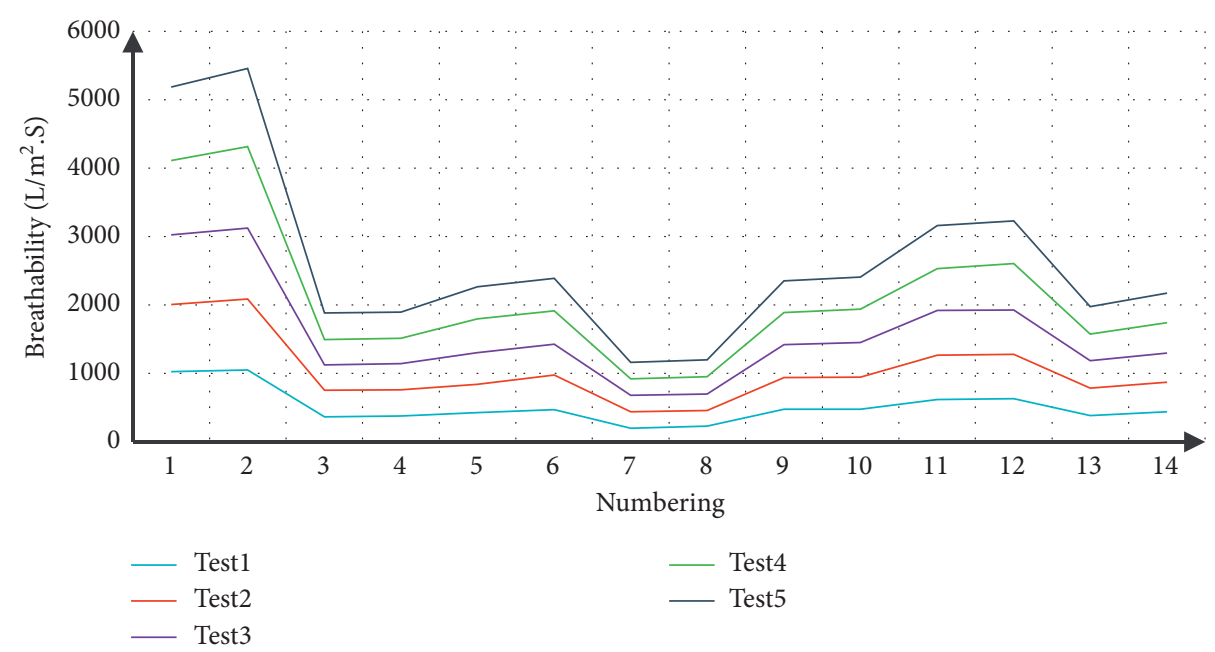

FIgURE 5: Air permeability comparison.

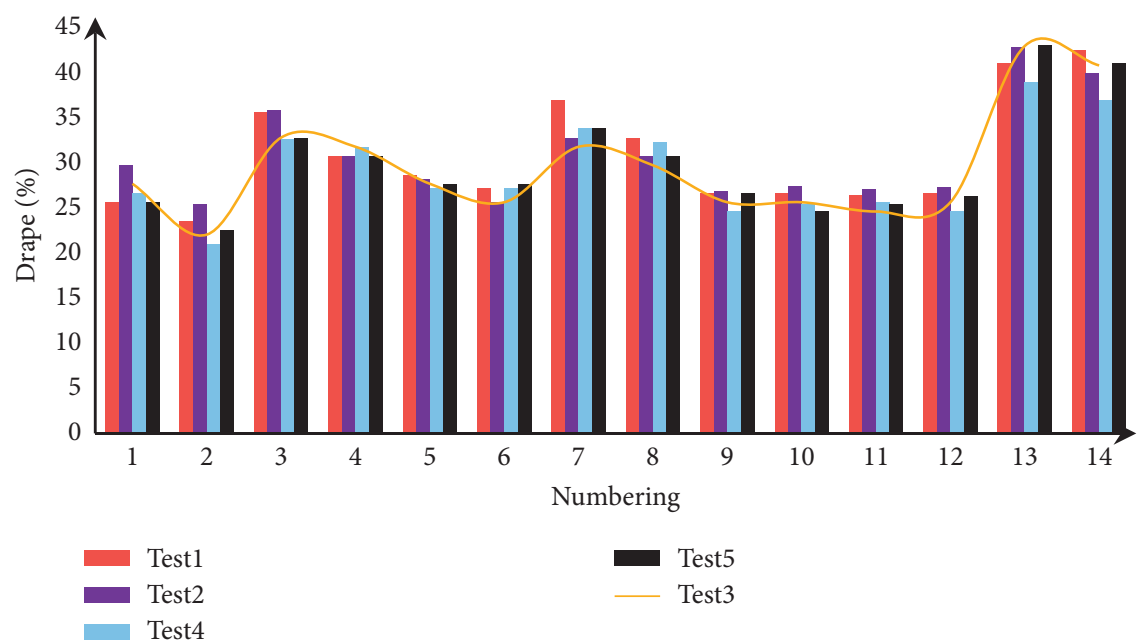

FIgURE 6: Drapability of fabric.

TABle 6: Reaction fabric strength.

\begin{tabular}{|c|c|c|c|c|c|c|}
\hline Sample & & 1 & 2 & 3 & 4 & 5 \\
\hline \multirow{2}{*}{ Unorganized } & Breaking strength $(N)$ & 402 & 412 & 431 & 421 & 359 \\
\hline & Stretching length $(\mathrm{mm})$ & 48 & 51 & 49 & 49 & 52 \\
\hline Organized & $\begin{array}{l}\text { Breaking strength }(N) \\
\text { Stretching length }(\mathrm{mm})\end{array}$ & $\begin{array}{c}432 \\
49\end{array}$ & $\begin{array}{c}445 \\
55\end{array}$ & $\begin{array}{c}434 \\
45\end{array}$ & $\begin{array}{c}459 \\
51\end{array}$ & $\begin{array}{c}467 \\
53\end{array}$ \\
\hline
\end{tabular}

TABLE 7: Different clothing properties.

\begin{tabular}{lcccc}
\hline Fabric color & Material & Organization & Weight & Quantity (piece) \\
\hline Light blue & Spandex + filament & $6+6$ rib & 302 & 136 \\
Dark camel & Filament & Jacquard & 226 & 1 \\
Red & Silk + cashmere & Flat needle & 266 & 1 \\
Blue & Spandex + spun silk & Jacquard & 256 & 2 \\
Color stripes & Spun silk & Flat needle & 189 & 2 \\
Green & Spun silk & Jacquard & 276 & 1 \\
Light stone cyan & Filament & Flat needle & 236 & 1 \\
Black & Spandex + spun silk & Flat stitch & 348 & 1 \\
Dark gray & Filament + spandex & Flat needle & & \\
\hline
\end{tabular}



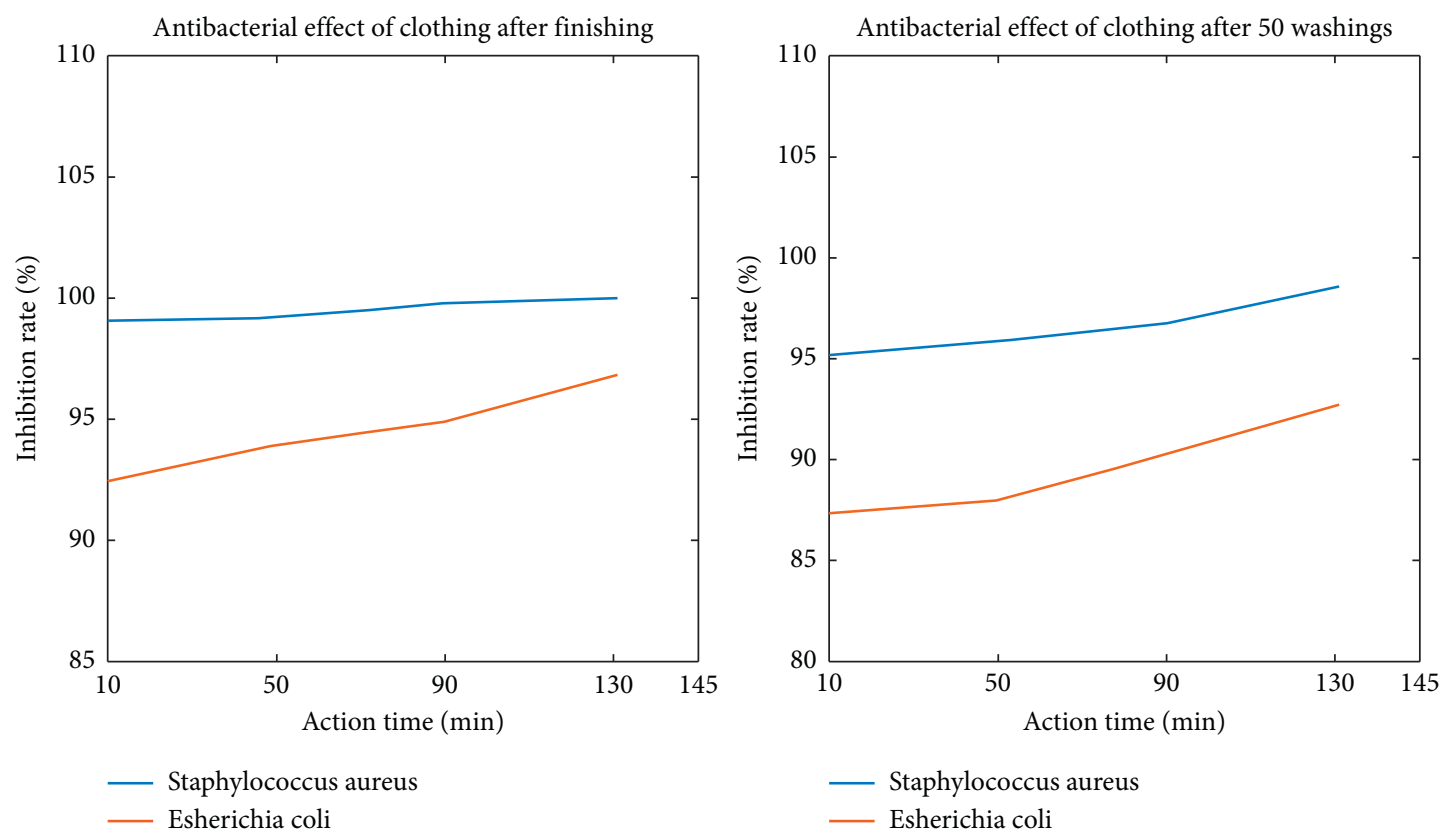

Figure 7: Nano-antimite and antibacterial knitted garment.

\section{Conclusion}

As a green and environmentally friendly photocatalyst, $\mathrm{TiO}_{2}$ has been used in air pollution treatment and water pollution treatment due to its stable chemical properties and low price. In addition, $\mathrm{TiO}_{2}$ is a kind of inorganic antibacterial agent; because of its long-lasting and broad-spectrum antibacterial properties, it does not easily produce drug resistance and has the advantages of thorough sterilization and no toxic side effects to the human body. It is used in the antibacterial finishing of textiles. In order to improve the photocatalytic performance of $\mathrm{TiO}_{2}$, this study has carried out sulfur and nitrogen codoping modification to prepare cotton fabrics loaded with doped $\mathrm{TiO}_{2}$ visible light catalytic durability antibacterial fabric, and its antibacterial properties were studied. This study involves the best antibacterial material, the best dispersant, the best dispersion process, the best finishing process, and the best plan considering the actual selection of the enterprise. Finally, the most effective antimite and antibacterial cotton knitted fabric is obtained. The results show that nano-Ag and $\mathrm{TiO}_{2}$ have outstanding antibacterial effect and the composite finishing agent is composed of photocatalysis and contact finishing agent, so its antibacterial effect is no longer affected by environmental conditions and will be more thorough. The best effect will be achieved when the ratio of $\mathrm{Ag}$ and $\mathrm{TiO}_{2}$ is $3: 1$. Dispersion is the key of nano-antimite and antibacterial finishing agent. In this study, through searching a large number of literature studies and referring to the research results of dispersion at home and abroad, the dispersion theory which can fully disperse nanoparticles was summarized. After a series of fabric mechanical properties and fabric style tests, this study revealed that cotton knitwear finished by nano-antimite and antibacterial finishing agent has good antimite and antibacterial effect and washing fastness. In future work, the dispersion problem is still the key of nanomaterials. The full dispersion of nanoparticles still needs to be further improved to make the obtained nanosolution more effective. Therefore, the dispersion process and finishing process still need to be further improved in the actual production of enterprises, so as to get the best antibacterial performance with the least investment.

\section{Data Availability}

The data that support the findings of this study are available from the author upon reasonable request.

\section{Conflicts of Interest}

The author declares no potential conflicts of interest with respect to the research, authorship, and/or publication of this article.

\section{References}

[1] B. Gao, N. Xu, and P. Xing, "Shock wave induced nanocrystallization during the high current pulsed electron beam process and its effect on mechanical properties," Materials Letters, vol. 237, no. 15, pp. 180-184, 2019.

[2] Z. Yanyan, W. Minghao, W. Mingjie, J. Zhu, and X. Zhang, "Multifunctional carbon-based nanomaterials: applications in biomolecular imaging and therapy," ACS Omega, vol. 3, no. 8, pp. 9126-9145, 2018.

[3] R. Paul, F. Du, L. Dai et al., "3D heteroatom-doped carbon nanomaterials as multifunctional metal-free catalysts for integrated energy devices," Advanced Materials, vol. 31, no. 13, Article ID 18055981, 2019.

[4] L. Li, C. Chen, H. Liu et al., "Multifunctional carbon-silica nanocapsules with gold core for synergistic photothermal and chemo-cancer therapy under the guidance of bimodal 
imaging," Advanced Functional Materials, vol. 26, no. 24, pp. 4252-4261, 2016.

[5] S. A. Santos, E. T. Silva, A. V. Caris, F. S. Lira, S. Tufik, and R. V. T. dos Santos, "Vitamin E supplementation inhibits muscle damage and inflammation after moderate exercise in hypoxia," Journal of Human Nutrition and Dietetics, vol. 29, no. 4, pp. 516-522, 2016.

[6] Y. Zhang, Y. Li, and C. Bai, "Microstructure and oxidation behavior of Si-MoSi 2 functionally graded coating on Mo substrate," Ceramics International, vol. 43, no. 8, pp. 6250-6256, 2017.

[7] Y. Zhang, W. Ni, and Y. Li, "Effect of siliconizing temperature on microstructure and phase constitution of Mo-MoSi2 functionally graded materials," Ceramics International, vol. 44, no. 10, pp. 11166-11171, 2018.

[8] L. Zhang, T. Liu, and Y. Chen, "Magnetic conducting polymer/mesoporous $\mathrm{SiO} 2$ yolk/shell nanomaterials: multifunctional nanocarriers for controlled release of doxorubicin," RSC Advances, vol. 6, no. 11, pp. 8572-8579, 2016.

[9] L. Qiu, Z. He, and D. Li, "Multifunctional cellular materials based on 2D nanomaterials: prospects and challenges," Advanced Materials, vol. 30, no. 4, pp. 17048501-170485015, 2018.

[10] G. Yang, H. Gong, T. Liu, X. Sun, L. Cheng, and Z. Liu, "Mesoporous silica-coated WS2@Fe3O4 nanosheets as a multifunctional theranostic nanocarrier for combination therapy of cancer," Nanomedicine: Nanotechnology, Biology and Medicine, vol. 12, no. 2, p. 523, 2016.

[11] N. Shadjou and M. Hasanzadeh, "Application of graphene and mesoporous silica nanomaterials on the orthopaedic implants: recent advances," Materials Technology, vol. 31, no. 13, pp. 806-811, 2016.

[12] Q. Ban, T. Bai, X. Duan, and J. Kong, "Noninvasive photothermal cancer therapy nanoplatforms via integrating nanomaterials and functional polymers," Biomaterials Science, vol. 5, no. 2, pp. 190-210, 2017.

[13] A. Peyvandi, I. Harsini, L. Ahmed Sbia et al., "Performance evaluation of precast concrete beams reinforced with multifunctional graphite nanomaterials," PCI Journal, vol. 61, no. 1, pp. 29-38, 2016.

[14] S. Raghav, R. Painuli, and D. Kumar, "Multifunctional nanomaterials for multifaceted applications in biomedical arena," International Journal of Pharmacology, vol. 13, no. 7, pp. 890-906, 2017.

[15] X. Li, T. Zhao, L. Sun et al., "The applications of conductive nanomaterials in the biomedical field," Journal of Biomedical Materials Research. Part A, vol. 104, no. 1, pp. 322-339, 2016.

[16] S. Marchesan, L. Ballerini, and M. Prato, "Nanomaterials for stimulating nerve growth," Science, vol. 356, no. 6342, pp. 1010-1011, 2017.

[17] C. Upendra, Q. Yiru, and J. F. Lovell, “Targeted nanomaterials for phototherapy," Nanotheranostics, vol. 1, no. 1, pp. 38-58, 2017.

[18] C.-F. He, C.-F. He, S.-H. Wang et al., "Advances in biodegradable nanomaterials for photothermal therapy of cancer," Cancer Biology \& Medicine, vol. 13, no. 3, pp. 299-312, 2016.

[19] T. C. Jackson, B. O. Patani, and M. B. Israel, "Nanomaterials and cell interactions: a review," Journal of Biomaterials and Nanobiotechnology, vol. 08, no. 4, pp. 220-228, 2017.

[20] Z. Zhang and M. R. King, "Nanomaterials for the capture and therapeutic targeting of circulating tumor cells," Cellular and Molecular Bioengineering, vol. 10, no. 1, pp. 275-294, 2017.
[21] L. Yuxuan, W. Lijun, L. Yuan et al., "Immunosensors based on nanomaterials for detection of tumor markers," Journal of Biomedical Nanotechnology, vol. 14, no. 1, pp. 44-65, 2018.

[22] X. Luo, W. Wang, J. R. Dorkin et al., "Poly(glycoamidoamine) brush nanomaterials for systemic siRNA delivery in vivo," Biomaterials science, vol. 5, no. 1, pp. 38-40, 2016.

[23] Y.-G. Bi, D. Di Liu, X.-M. Liu, and S.-Q. Zhou, "Adsorption of $\mathrm{Cu} 2+$ from aqueous solution using PEG modified hydroxyapatite nanomaterials as adsorbent," Journal of Biobased Materials and Bioenergy, vol. 11, no. 4, pp. 364-371, 2017.

[24] T. M. Becker, J. Heinen, D. Dubbeldam, L.-C. Lin, and T. J. H. Vlugt, "Polarizable force fields for $\mathrm{CO} 2$ and $\mathrm{CH} 4$ adsorption in M-MOF-74," Journal of Physical Chemistry C, vol. 121, no. 8, pp. 4659-4673, 2017.

[25] T. Inprasit, K. Motina, P. Pisitsak, and P. Chitichotpanya, "Dyeability and antibacterial finishing of hemp fabric using natural bioactive neem extract," Fibers and Polymers, vol. 19, no. 10, pp. 2121-2126, 2018.

[26] N. A. Raouf, N. M. A. Enazi, I. B. M. Ibraheem, R. M. Alharbi, and M. M. Alkhulaifi, "Antibacterial and anti-hyperlipidemic activities of the green alga Cladophora koeiei," Beni-Suef University Journal of Basic and Applied Sciences, vol. 7, no. 1, pp. $158-164,2018$.

[27] C. H. Lim, K. Kim, and Y. Cheong, "Factors affecting sportswear buying behavior: a comparative analysis of luxury sportswear," Journal of Business Research, vol. 69, no. 12, pp. 5793-5800, 2016.

[28] B. Woo-Yeul, K. K. Byon, C. Young-Hwan, and C. W. Park, "Millennial consumers' perception of sportswear brand globalness impacts purchase intention in cause-related product marketing," Social Behavior \& Personality An International Journal, vol. 45, no. 8, pp. 1319-1335, 2017.

[29] D. Baumann-Pauly, A. G. Scherer, and G. Palazzo, "Managing institutional complexity: a longitudinal study of legitimacy strategies at a sportswear brand Company," Journal of Business Ethics, vol. 137, no. 1, pp. 31-51, 2016.

[30] J. T. Kalkhoven and M. L. Watsford, "The relationship between mechanical stiffness and athletic performance markers in sub-elite footballers," Journal of Sports Sciences, vol. 36, no. 2, pp. 1022-1029, 2018.

[31] J. Drager, J. Rasio, and A. Newhouse, "Athletic pubalgia (sports hernia): presentation and treatment," Arthroscopy: The Journal of Arthroscopic \& Related Surgery, vol. 36, no. 12, pp. 2952-2953, 2020.

[32] T. A. Hanson, M. R. Bryant, and K. J. Lyman, "Intercollegiate athletic programs, university brand equity and student satisfaction," International Journal of Sports Marketing \& Sponsorship, vol. 21, no. 1, pp. 106-126, 2019. 